\title{
Hypoxia in the Neoplastic Context
}

\section{Introduction}

Cancer is one of the leading causes of death worldwide, with malignancies of the lungs leading the neoplastic rank, followed by colon, rectum, and breast. ${ }^{1}$ Treatment is estimated to cost the NHS and private funding around $£ 9.4$ billion per year. This means that an average of $£ 30,000$ is assigned to a single person in a year. ${ }^{2}$ With 350000 new cases diagnosed each year in the UK and 150000 cancerrelated deaths, ${ }^{3}$ understanding the pathogenesis and investigating potential targets for treatment is of paramount importance.

In this essay I will discuss the existing link between hypoxia and more aggressive phenotypes of tumours as well as the poorer prognosis associated with this phenomenon, ${ }^{4}$ exploring the molecular processes that underlie this association. In particular, Hypoxia Inducible Factor pathway will be analysed.

\section{Hypoxia in the neoplastic context}

Oxygen is needed by human cells in order to achieve aerobic metabolism and obtain efficient amounts of energy in the form of ATP for life. Hypoxia (non-physiological level of oxygen tension) jeopardises this process and may lead to serious cell and tissue damage. Through evolution, adaptive mechanisms have emerged that allow not only to alter cell metabolism, but to also create changes in the cellular surroundings in response to hypoxia.

Hypoxia is a common scenario in the micro-environment of malignancy. ${ }^{5}$ This can be easily understood when appreciating the imbalance between supply and demand of oxygen that hyperproliferation of cells creates in tumour tissue, where more cells demanding oxygen are faced with a greater distance to the existing vasculature, hence hampering oxygen diffusion. When a cell is exposed to hypoxia, a vast number of processes commence in the form of complex intracellular pathways. Some lead to modulation of cell metabolism, proliferation, survival, apoptosis, inflammation, and metastasis in the context of cancer. The most important pathways in this setting are PI3K/AKT/mTOR/MAPK, NF- $\mathrm{KB}$ pathways, and most relevantly, the Hypoxia Inducible Factor pathway (HIF-1). ${ }^{6}$

\section{What is HIF-I?}

HIF-1 is a heterodimeric protein complex that serves as a transcriptional factor. It is composed by a constitutively expressed $\beta$ subunit and an oxygen regulated $\alpha$ subunit (HIF- $1 \alpha$, expressed in all cells; HIF-2 $\alpha$ which is only expressed in certain types of cells such as endothelial cells; and HIF-3 $\alpha$ of which profile of expression and function are largely unknown $)^{7}$ which is constantly degraded at a very fast rate (half life of approximately 5 minutes) ${ }^{8}$ by hydroxylation, catalysed by the PHD and FIH-1 enzymes, both of which are upstream of HIF-1 and oxygen-dependent for activity. ${ }^{9}$ In hypoxic conditions, these enzymes lose their activity and a rise of HIF-1 $\alpha$ subunit concentration follows. When this dimerises with the HIF- $1 \beta$ subunit through their basic helix-loop-helix domain to form a heterodimeric complex, the complete HIF-1 heterodimer binds to hypoxia response elements (HREs) in the genome to initiate the transcription of the adaptive pathway. ${ }^{10} \mathrm{~A}$ relation can be established between
Volume 9 Issue I - 2019

\author{
Pablo Martínez-Soler,' Juan F Martínez- \\ Canca $^{2}$ \\ 'Medical Student, University of Aberdeen, UK \\ ${ }^{2}$ Consultant Neurosurgeon, Chip Hospital, Spain
}

\begin{abstract}
Correspondence: Dr Juan F Martínez-canca, MD, MRCS, Consultant Neurosurgeon, Head of Service Chip Hospital, Málaga, Spain, Tel +447595368967,

Email pablom@doctor.com
\end{abstract}

Received: August 04, 2018 | Published: January 09, 2019

concentration of HIF- $1 \alpha$ subunits and tumour aggressiveness. In the following section I will explore this relation by shedding some light on the underlying processes (Figure 1).

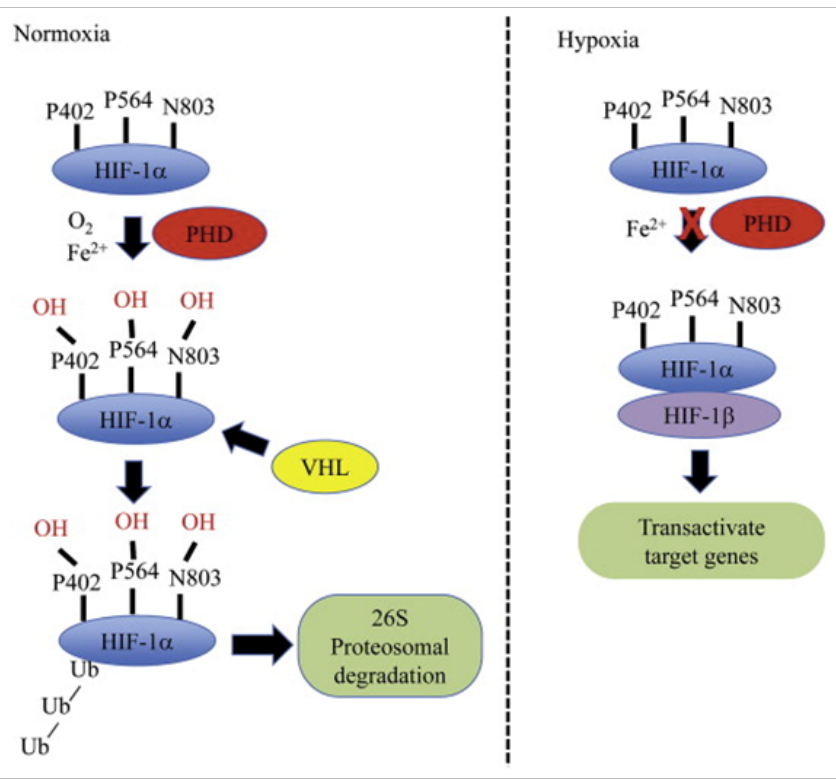

Figure I Overview of oxygen-dependent regulation of HIF-I $\alpha . "$

\section{How does hif-I make tumours more aggressive?}

HIF-1 dimerisation in tumour cells results in the tumour manifesting a more aggressive phenotype. This is achieved through the transcriptional activation of over 100 genes in charge of regulating biological processes required for tumour survival. ${ }^{12}$ Activation of these genes prompts responses such as enhancing cell survival, inhibition of pro-apoptotic pathways, cell metabolism changes, neovascularization, and induction of cell migration and invasion, resulting in the expression of attributes linked to aggressiveness. Likewise, raised HIF- $1 \alpha$ concentrations are behind relevant events that lead to an increased resistance to chemotherapy and radiotherapy (Figure 2). ${ }^{13,14}$ 


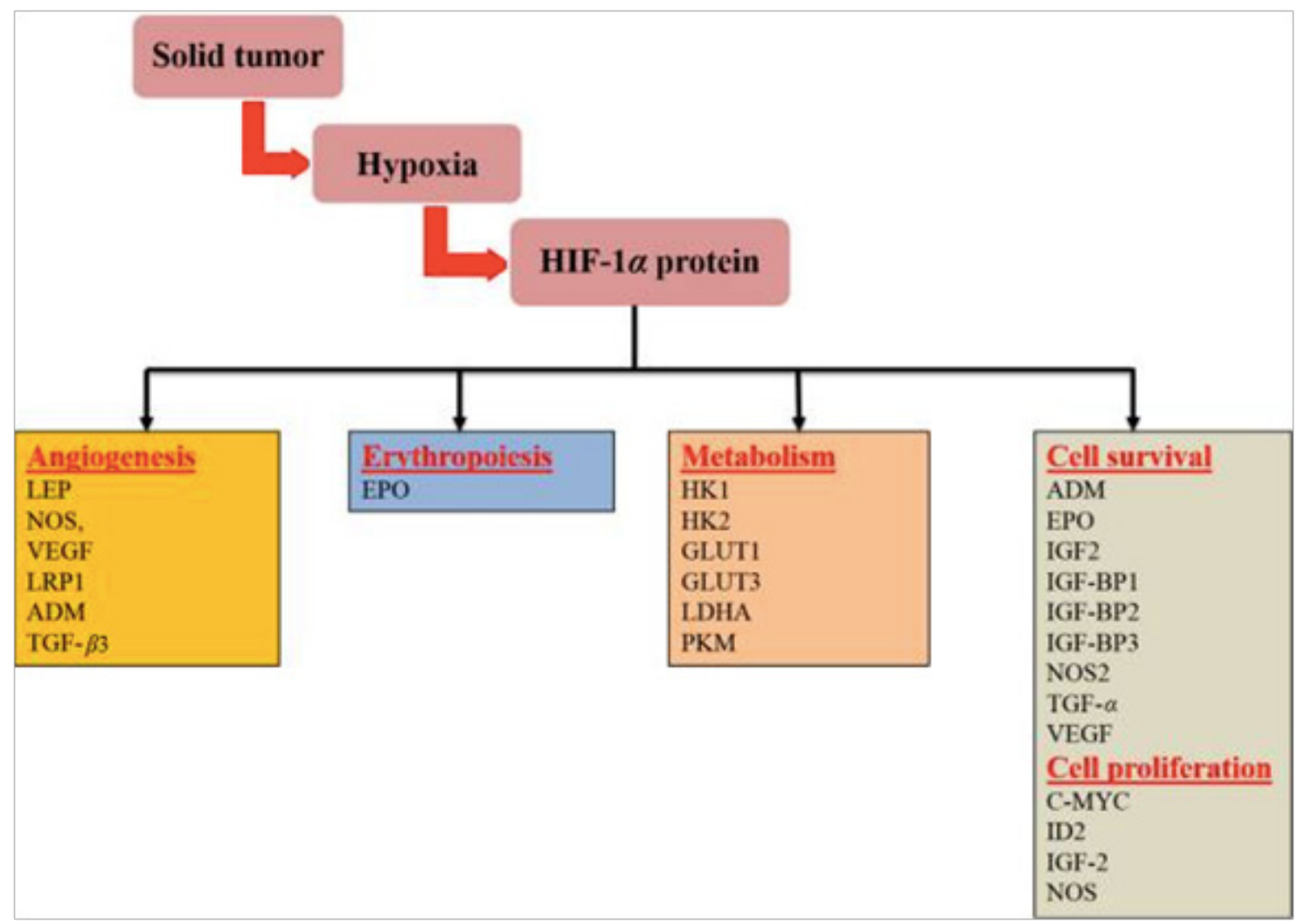

Figure 2 Representative HIF- I $\alpha$ regulatory genes and their effects on cancer progression. ${ }^{14}$

\section{Vascular changes}

One of the central aspects of tumour aggressiveness promoted by HIF-1 is its characteristic neovascularisation. In normal conditions, assuming embryological development as an example, the growth of new vessels (vasculogenesis) involves bone marrow derived endothelial progenitor cells. ${ }^{15}$ These primitive vessels will generate new ones by angiogenesis. ${ }^{16}$ In the final step of their development, these new vessels, composed of endothelial cells joined by tight junctions interact with smooth muscle cells and pericytes (maturation).

In the scenario of hypoxic tumour tissue, hypoxia and HIF-1 $\alpha$ subunits have been shown to contribute in every step of new vessel formation in three main ways. By regulation of VEGF (Vascular Endothelial Growth Factor) they induce differentiation of endothelial progenitor cells (which they also aid to recruit from bone marrow) into endothelial cells. Angiogenesis is also supported by the transcriptional expression of enzymes that sprout the already existing vasculature. These new vessels that have yet to mature allow endothelial cells to migrate across the extra-cellular matrix in response to chemoattractants. Hypoxia and HIF-1 will then induce their (endothelial cells) proliferation through the regulation of VEGF-R1 (Flt-1), Ang-1 and Ang-2 expression. ${ }^{17}$ The final step, maturation of the vessels, is also facilitated by hypoxia and HIF-1 by induction of Ang-1, PDGF, and TGF- $\beta$ that complete and stabilise these new vessels by recruiting the aforementioned smooth muscle cells and pericytes. ${ }^{15}$ However, due to the nature of malignant neoplasias, when new blood supply is established, cells will thrive swiftly and demand oxygen, returning the tumour microenvironment to its hypoxic condition and, in turn, activating the previously mentioned neovascularising mechanisms. This means that tumours will usually have a rich, extensive, yet flawed vascularisation. ${ }^{18}$

\section{Metabolism changes}

Hypoxic tumour cells undergo metabolic adaptations in order to survive. These adaptations make the cell less glucose efficient to the point where even under normal oxygen conditions they will use more glucose to produce lactic acid than normal cells. ${ }^{19}$ This is named the Warburg effect, in honour of its first observer and Nobel laureate Otto Warburg. The German physiologist described how cancer cells switch their source of ATP production from the aerobic tricarboxylic acid cycle and oxidative phosphorylation in the mitochondria to cytoplasmic glycolysis: significantly less efficient. Further research has demonstrated that there are many cancer genes such as Ras, $\mathrm{cMyc}$, and p53 that have a role in oncogenic metabolic alterations. ${ }^{20}$ As one of the main regulators of hypoxic response in cancer, HIF-1 protects cancer cells from hypoxic stress by mediating their metabolic regulations in what has recently been discovered to be a feed-forward mechanism, with HIF-1 $\alpha$ targets such as glycolytic enzyme pyruvate kinase M2 (PKM2) enhancing HIF-1 $\alpha$ binding to hypoxia response elements (HRE). ${ }^{21}$

A "hot area" in a PET scan may be indicative of active malignancy. This is a reflection of the abundant use of glucose (Fludeoxyglucose marker in PET scans) by cancer cells under the Warburg effect. HIF-1 facilitates the expression of GLUT-1 and GLUT-3, insulin independent membrane glucose transporters, to allow the increased glucose uptake this metabolic adaptation requires. ${ }^{22}$ Observing normal physiology, when glucose enters the cell it is phosphorylated to maintain a concentration gradient for glucose uptake, it is then subjected to the action of glycolytic enzymes. The enzymes responsible for this process, hexokinases (HK1 and HK2) and phosphoglycerate kinase 1 (PGK1), are also induced by HIF-1. ${ }^{23}$ Finally, HIF-1 has also been shown to promote lactate production and electron acceptor 
$\mathrm{NAD}+$ generation by up-regulating lactate dehydrogenase A (LDHA) expression. ${ }^{24}$

HIF-1 is not only in charge of regulating the metabolic shift to cytoplasmic glycolysis, it is also responsible for the inhibition of the Krebs Cycle and oxidative phosphorylation pathways in the mitochondria. HIF- $1 \alpha$ promotes activity of the pyruvate dehydrogenase kinase isozyme 1 (PDK1), an enzyme that inhibits pyruvate dehydrogenase (PDH) by phosphorylating its serine residues at 3 sites. ${ }^{25}$ As a consequence, PDH catalysed conversion of Pyruvate to Acetyl-CoA is down-regulated, further contributing to the metabolic adaptation to hypoxia driven by HIF-1 (Figure 3).

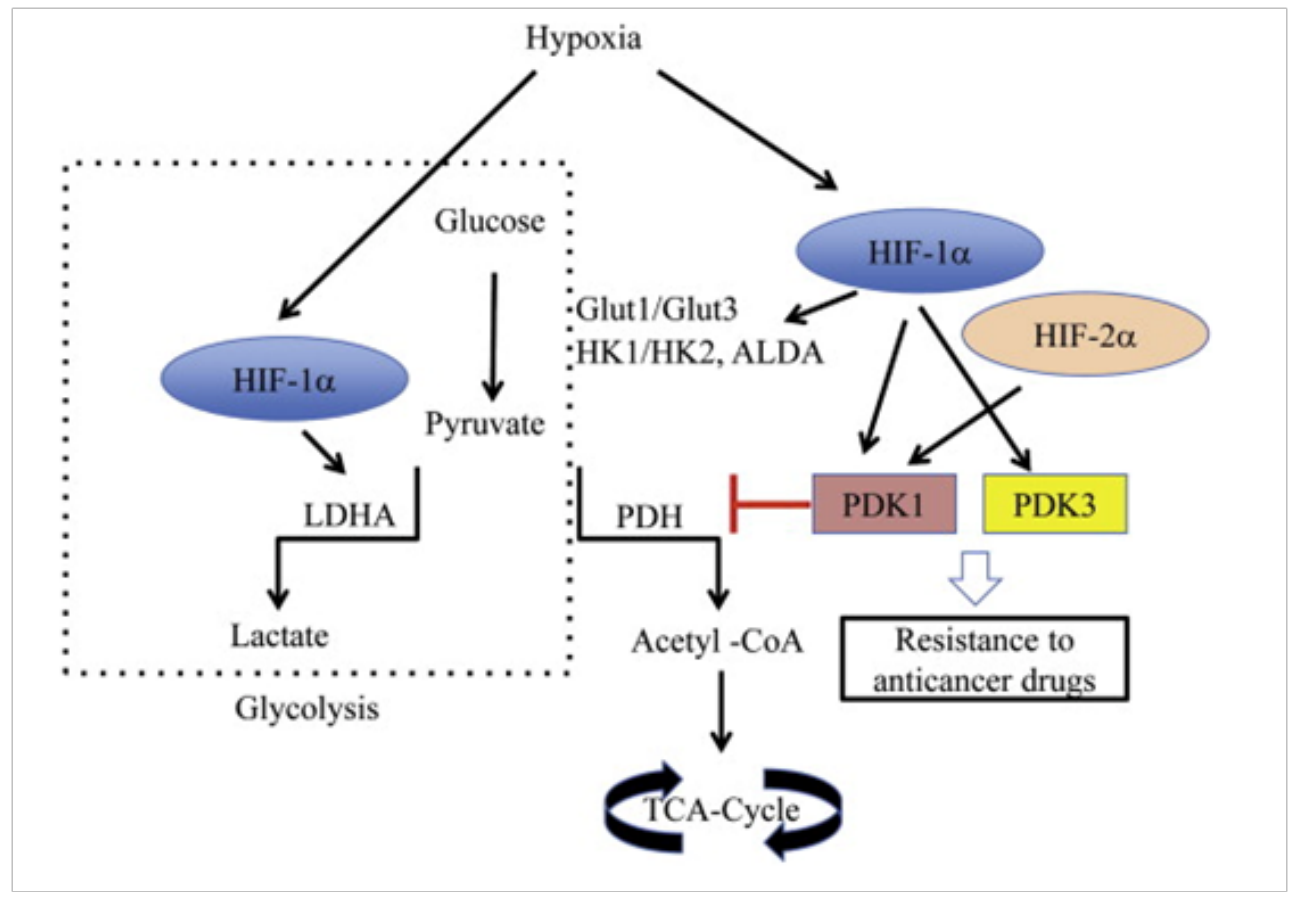

Figure 3 Broad overview of the regulation of metabolism by HIF-I $\alpha .{ }^{.1}$

\section{Inhibition of proapoptotic pathways}

Although the exact mechanisms under which apoptosis is regulated in hypoxia are not completely understood, hypoxia has been found to increase the transcriptional activity of anti-apoptotic genes such as IAP-2, Bcl-2 and Bcl-XL, ${ }^{26}$ to activate the PI-3k/Akt survival pathway, ${ }^{27}$ and to down-regulate activity of $\mathrm{p} 53$, a protein with strong anti-tumour properties activated in cases of DNA damage that usually stimulates cell cycle arrest or apoptosis ${ }^{28}$ (it has been nicknamed "the guardian of the genome"), via over-expression of MDM $2,{ }^{29}$ the ubiquitin ligase that mediates p53 degradation. Hypoxia also blocks the translocation of apoptosis regulator BCL2 associated $\mathrm{X}$ protein $(\mathrm{BAX})$ to the mitochondria, where it acts as an essential signalling molecule for apoptosis (opening of voltage-dependent anion channels, loss of membrane potential, release of cytochrome c...) further allowing the avoidance of apoptosis by cancer cells. ${ }^{30}$ Research has also demonstrated the involvement of HIF- $1 \alpha$ in transcription of the promoter region of telomerase genes hTERT and hTR, ${ }^{31}$ effectively expanding the life of malignant cells beyond the Hayflick limit (maximum number of times a cell can divide) by conserving telomere length.

\section{Increased metastasis rates}

As mentioned previously, one of the main factors contributing to the poor prognosis associated to hypoxic tumours is the increased incidence of metastasis. This phenomenon, of a marked mechanical quality, is tightly related to the aforementioned neovascularisation seen in this scenario: a profuse yet faulty network of permeable capillaries that facilitates extravasation and relocation of cancer cells in other environments. Acting synergistically with this, both hypoxia and HIF-1 $\alpha$ overexpression grant cells with mobility and invasive characteristics by promoting epithelial-mesenchymal transition (EMT), a differentiation of cell morphology and gene expression that results in a down-regulation of transmembrane molecules such as E-cadherin. ${ }^{32}$ Additionally, hypoxia increases the invasiveness of tumours both through blood/lymph vessels and through the basement membrane: In the former, this is achieved by altering the interactions between components of the extracellular matrix and up-regulation of urokinase-type plasminogen activator receptor (UPAR) $)^{33}$ which increases proteolytic activity. For the latter, extravasation through blood/lymph vessels, hepatocyte growth factor (HGF)-MET provides the cells with a migratory tendency towards these structures (Figure 4). ${ }^{34}$

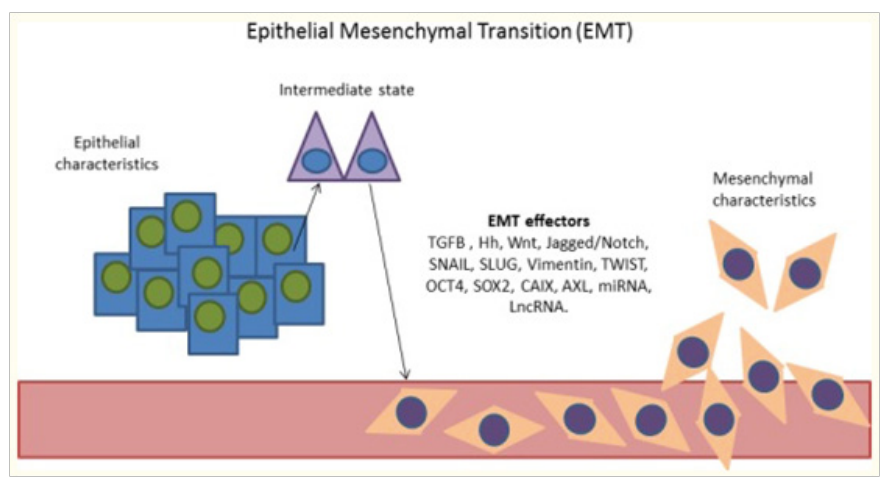

Figure 4 EMT influenced spread. ${ }^{42}$ 


\section{Hypoxia and increased resistance to treatment}

The immune system is, after DNA repairing mechanisms, one of the first defence lines against cancer. In hypoxic setting, the expression of HIF-1 results in tumours attracting macrophages, which mature into tumour associated macrophages (TAM). By the action of hypoxia-induced immunosuppressive factors such as IL-10 and prostaglandins, TAMs are rendered unable to phagocytise dead cells or to present antigens to T-cells, ${ }^{35}$ who in these conditions are prevented from initiating activation-induced cell death. ${ }^{36}$ Hence, in the presence of hypoxia and HIF-1, tumours remain "under the radar".

Radiotherapy has long been known to be two and even three times less effective under hypoxic conditions when compared to normoxic cells exposed to the same treatment. ${ }^{37}$ The explanation lies in its mechanism of action, that aims to destroy the DNA of targeted cells by forming oxygen-derived free hydroxyl radicals when it interacts with intracellular water and to stabilise these radicals to prevent DNA repair. This capacity is significantly reduced in hypoxia.

When it comes to chemotherapy, the main issue encountered in hypoxic conditions is the cell cycle arrest or autophagy states promoted by HIF-1 $\alpha$ that cells enter to maintain homeostasis under stress $^{38}$ and the influence of the HIFs that inhibits p 53 normal apoptotic process, ${ }^{39}$ a significant factor in cancer drug resistance. Even though the complete mechanisms behind these processes are unknown, it is acknowledged that clinically used agents such as tyrosine inhibitors become incompetent under hypoxic conditions. ${ }^{40}$ Research has also shown that HIF-1 inhibition reverses multi-drug resistance through down-regulation of MDR1/P-glycoprotein and increases tumour sensitivity to radiotherapy. ${ }^{41}$ A significant amount of hypoxia-induced treatment resistance obeys a similar rationale, such as resistance to vinca-alkaloids and methotrexate, mediated by HIF-1s influence over cell cycle arrest and p53, for which G1/S-phase arrest is liable. ${ }^{39}$ Additionally, aberrant vascularisation and extracellular acidosis have been shown to hamper therapeutic agent diffusion, their intracellular concentrations (anthracyclines, bleomycin), and their very action (vinblastine, doxorubicin). ${ }^{42}$

\section{A glance into the future: Hypoxia as a treatment target}

As we continue to learn about the pathways involved in hypoxia response, new potential targets for treatment emerge and effective medical care for cancer shapes itself in ways never even dreamt of by past generations of physicians. Drugs that target elements mentioned in this essay are already in clinical use (VEGF inhibitors, radiosensitizers, hyperbaric oxygen...). Open research fronts are abundant and possibilities are endless. Regarding hypoxia, some of the latest developments are:

\section{i. Targeting HIF-1 $\alpha$}

One of the most advanced novel HIF-1 $\alpha$ inhibitors is Digoxin. It has been shown to inhibit HIF-1 $\alpha$ synthesis and, after proving effective in vitro and in vivo, is set to finish phase II clinical trials in July $2018 .{ }^{43}$

\section{ii. Inhibiting HIF-1 $\alpha$ targets}

Hepatocyte growth factor (HGF)-MET pathway, induced by HIF$1 \alpha$ (mentioned previously when discussing EMT), is a promising treatment target due to its involvement in metastasis and VEGF inhibitor resistance. ${ }^{44}$ Combination of Crizotinib (MET and ALK inhibitor) and Axitinib (selective tyrosine kinase inhibitor of VEGF receptor 1,2, and 3), is currently in phase I trial, the first of its kind to combine both agents, scheduled for completion on 31 July of $2018 .{ }^{45}$

\section{iii. Targeting metabolic changes}

From this investigation line, combination of anti-bacterial agents (doxycycline, paramomycin, clarithromycin, clindamycin, dapsone, and miltefosine) alone or along anti-fungal agents is the most interesting approach due to two factors. Firstly, they are all well known agents that are simply being used for a different commitment (targeting mitochondria for anti-cancer purposes). Secondly, this treatment is aimed at patients with untreatable disease, yet expects tumour regression at 6 months. Trial is estimated to conclude in December 2020 (Figure 5). ${ }^{46}$

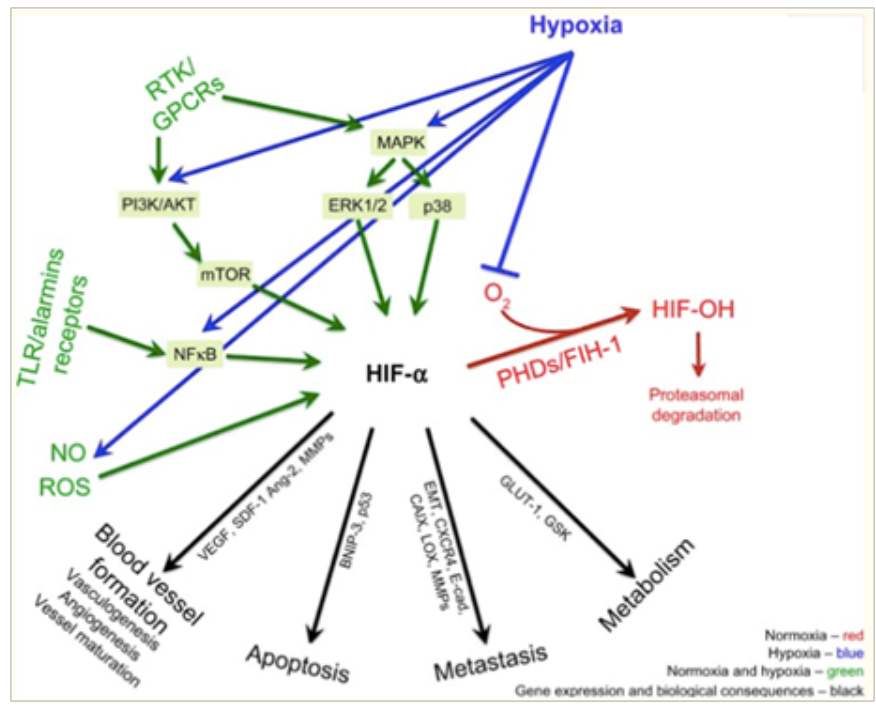

Figure 5 Broad overview of Hypoxia and HIF-I effects. ${ }^{47}$

\section{Conclusion}

In conclusion, one can appreciate how the hypoxic microenvironment in which tumours thrive incites the surge of more aggressive phenotypes that will further contribute to the sustainment of hypoxic and acidic conditions. Importance must continue to be given to understanding this vast milieu, these circumstances, and the numerous cellular mechanisms that interact constantly so that we can progress in the development of effective treatment.

\section{Acknowledgments}

None.

\section{Conflicts of interest}

The author declares no conflicts of interest.

\section{References}

1. World Health Organization. Cancer. 2018.

2. nhs.uk. Cancer survival rates 'threatened by rising cost'. World Health Organization. 2018.

3. Cancer Research UK. Cancer Statistics for the UK. 2018.

4. Welsh SJ, Powis G. Hypoxia Inducible Factor as a Cancer Drug Target. Current Cancer Drug Targets. 2003;3(6):391-405.

5. Semenza GL. Defining the role of hypoxia-inducible factor 1 in cancer biology and therapeutics. Oncogene. 2010;29(5):625-634. 
6. Seta KA, Spicer Z, Yuan Y, et al. Responding to Hypoxia: Lessons From a Model Cell Line. Science Signaling. 2002;2002(146):re11.

7. Huang D, Li C, Zhang H. Hypoxia and cancer cell metabolism. Acta Biochimica et Biophysica Sinica. 2014;46(3):214-219.

8. Salceda S, Caro J. Hypoxia-inducible Factor $1 \alpha(\mathrm{HIF}-1 \alpha)$ Protein Is Rapidly Degraded by the Ubiquitin-Proteasome System under Normoxic Conditions. J Biol Chem. 1997;272(36):22642-22647.

9. Wang GL, Jiang BH, Rue EA. Hypoxia-inducible factor 1 is a basic-helix-loop-helix-PAS heterodimer regulated by cellular $\mathrm{O} 2$ tension. Proc Natl Acad Sci U S A. 1995;92(12):5510-5514.

10. Kaelin WG Jr, Ratcliffe PJ. Oxygen Sensing by Metazoans: The Centra Role of the HIF Hydroxylase Pathway. Mol Cell. 2008;30(4):393-402.

11. Liao W, Lin S, Sunny Sun H, et al. Hypoxia-induced tumor malignancy and drug resistance: Role of microRNAs. Biomarkers and Genomic Medicine. 2014;6(1):1-11.

12. Schödel J, Oikonomopoulos S, Ragoussis J, et al. High-resolution genome-wide mapping of HIF-binding sites by ChIP-seq. Blood. 2011;117(23):e207-e217.

13. Semenza G. HIF-1 and mechanisms of hypoxia sensing. Curr Opin Cell Biol. 2001;13(2):167-171.

14. Masoud GN, Li W. HIF-1 $\alpha$ pathway: role, regulation and intervention for cancer therapy. Acta Pharmaceutica Sinica B. 2015;5(5):378-389.

15. Conway EM, Collen D, Carmeliet P. Molecular mechanisms of blood vessel growth. Cardiovasc Res. 2001;49(3):507-521.

16. Carmeliet P. Angiogenesis in life, disease and medicine. Nature. 2005;438(7070):932-936.

17. Krock BL, Skuli N, Simon MC. Hypoxia-Induced Angiogenesis: Good and Evil. Genes Cancer. 2011;2(12):1117-1133.

18. Carmeliet P, Jain RK. Molecular mechanisms and clinical applications of angiogenesis. Nature. 2011;473(7347):298-307.

19. Warburg O. On the origin of cancer cells. Science. 1956;123(3191):309314

20. Dang CV, Semenza GL. Oncogenic alterations of metabolism. Trends Biochem Sci. 1999;24(2)68-72.

21. Luo WB, Hu HX, Chang R, et al. Pyruvate kinase M2 is a PHD3-stimulated coactivator for hypoxia-inducible factor 1. Cell. 2011:145(5):732-744.

22. Gordan JD, Thompson CB, Simon MC. HIF and c-Myc: sibling rivals for control of cancer cell metabolism and proliferation. Cancer Cell. 2007;12(2):108-113.

23. Mathupala SP, Rempel A, Pedersen PL. Glucose catabolism in cancer cells: identification and characterization of a marked activation response of the type II hexokinase gene to hypoxic conditions. J Biol Chem. 2001;276(46):43407-43412.

24. Semenza GL, Jiang BH, Leung SW, et al. Hypoxia response elements in the aldolase A, enolase 1, and lactate dehydrogenase A gene promoters contain essential binding sites for hypoxia-inducible factor 1. J Bio Chem. 1996:271(51):32529-32537.

25. Kim JW, Tchernyshyov I, Semenza GL, et al. HIF-1-mediated expression of pyruvate dehydrogenase kinase: a metabolic switch required for cellular adaptation to hypoxia. Cell Metab. 2006;3(3):177-185.

26. Ruan K, Song G, Ouyang G. Role of hypoxia in the hallmarks of human cancer. J Cell Biochem. 2009:107(6):1053-1062.

27. Zhou J, Schmid T, Schnitzer S, et al. Tumor hypoxia and cancer progression. Cancer. 2006;237(1):10-21.
28. Lozano G. The oncogenic roles of p53 mutants in mouse models. Curr Opin Genet Dev. 2007;17(1):66-70.

29. Kim M, Park SY, Pai HS, et al. Hypoxia inhibits tumor necrosis factorrelated apoptosis-inducing ligand-induced apoptosis by blocking Bax translocation. Cancer Res. 2004;64(12):4078-4081.

30. Kim M, Park SY, Pai HS, et al. Hypoxia inhibits tumor necrosis factorrelated apoptosis-inducing ligand-induced apoptosis by blocking Bax translocation. Cancer Res. 2004;64(12):4078-4081.

31. Seimiya H, Tanji M, Oh-hara T, et al. Hypoxia up-regulates telomerase activity via mitogen-activated protein kinase signaling in human solid tumor cells. Biochem Biophys Res Commun. 1999;260(2):365-370.

32. Thiery JP, Sleeman JP. Complex networks orchestrate epithelialmesenchymal transitions. Nat Rev Mol Cell Biol. 2006;7(2):131-142.

33. Sullivan R, Graham CH. Hypoxia-driven selection of the metastatic phenotype. Cancer Metastasis Rev. 2007;26(2):319-331.

34. Chan DA, Giaccia AJ. Hypoxia, gene expression, and metastasis. Cancer Metastasis Rev. 2007;26(2):333-339.

35. Talks KL, Turley H, Gatter KC, et al. The expression and distribution of the hypoxia-inducible factors HIF-1alpha and HIF-2alpha in normal human tissues, cancers, and tumor-associated macrophages. Am J Pathol. 2000;157(2):411-421.

36. Sitkovsky M, Lukashev D. Regulation of immune cells by local-tissue oxygen tension: HIF1 alpha and adenosine receptors. Nat Rev Immunol. 2005;5(9):712-721.

37. Rockwell S, Dobrucki IT, Kim EY, et al. Hypoxia and Radiation Therapy: Past History, Ongoing Research, and Future Promise. Current Molecular Medicine. 2009;9(4):442-458.

38. Notte A, Ninane N, Arnould T, et al. Hypoxia counteracts taxol-induced apoptosis in MDA-MB-231 breast cancer cells: role of autophagy and JNK activation. Cell Death Dis. 2013;4:e638.

39. Maddika S, Ande SR, Panigrahi S, et al. Cell survival, cell death and cell cycle pathways are interconnected: implications for cancer therapy. Drug Resist Updat. 2007;10(1-2):13-29.

40. Ahmadi M, Ahmadihosseini Z, Allison SJ, et al. Hypoxia modulates the activity of a series of clinically approved tyrosine kinase inhibitors. $\mathrm{Br} \mathrm{J}$ Pharmacol. 2013;171(1):224-236.

41. Chen J, Ding Z, Peng Y, et al. HIF-1 $\alpha$ Inhibition Reverses Multidrug Resistance in Colon Cancer Cells via Downregulation of MDR1/PGlycoprotein. PLoS ONE. 2014;9(6):e98882.

42. Adamaki M, Georgountzou A, Moschovi M. Cancer and the Cellular Response to Hypoxia. Pediatr Therapeut. 2013;S1:002.

43. Newman RA, Yang P, Pawlus AD, et al. Cardiac Glycosides as Novel Cancer Therapeutic Agents. Mol Interv. 2008;8(1):36-49.

44. Paolicchi E, Gemignani F, Krstic-Demonacos M, et al. Targeting hypoxic response for cancer therapy. Oncotarget. 2016;7(12): 13464-13478.

45. Clinicaltrials.gov. A Phase 1b Study of axitinib in combination with crizotinib in patients with advanced solid tumors. (2018).

46. Clinicaltrials.gov. Clinical Evaluation of a New Form of Cancer Therapy Based on the Principles of Atavistic Metamorphosis (Atavistic Chemotherapy). 2018.

47. Muz B, de la Puente P1, Azab F, et al. The role of hypoxia in cancer progression, angiogenesis, metastasis, and resistance to therapy. Hypoxia. 2015;3:83-92. 